\title{
Soil Physics Teaching Tools: Steady-State Water Movement in Soils
}

\author{
David L. Nofziger* and Jinquan Wu
}

\begin{abstract}
Understanding water movement in soils is essential for understanding other soil physical processes. This generally means the introduction of Darcy's equation for saturated systems and the Darcy-Buckingham equation for unsaturated soils. Although these equations look relatively simple, they can be complex and their predictions are not always intuitive. Solving these equations can be difficult for many conditions; therefore, many aspects of water flow are presented qualitatively or omitted in texts and classes. We developed interactive Java applets that allow users to specify soil hydraulic properties and to define flow systems of interest. The software calculates the steady-state rate of water movement in those systems and displays graphs of total, matric, or gravitational potential, hydraulic conductivity, driving force, or water content in the soil as functions of position. The applets can be run from common Web browsers. An instructor can use the software to demonstrate principles while lecturing, or incorporate it into assignments, educational material, and examinations. Since the tools are available on the Web, users can access supplemental material that explains concepts. Moreover, the applets can be incorporated into Web-based decision-support systems to enhance a decision-maker's understanding of these processes. Numerical experiments utilizing the applets are available. These tools enable users to discover flow principles, calculate flow in specific systems, and gain insight into the sensitivity of water movement to hydraulic parameters needed to describe soils and flow systems. They provide a visual means of comprehending these abstract equations and the complex manner in which water moves through soils.
\end{abstract}

$\mathrm{D}$ ARCY'S EQUATION is one of the most fundamental equations in soil physics. It is the foundation for the modern study of water and chemical movement in soils. In 1856, Henri Darcy formulated the equation based on his classic investigation of seepage rates through sand filters in the city of Dijon, France. Since then, many experiments have been carried out to test and to expand the equation and to characterize the required hydraulic properties of different soils. Slichter (1899) generalized the mathematical expression of Darcy to describe multidimensional flow through saturated porous media. Buckingham (1907) extended Darcy's equation for water flow in a rigid, unsaturated porous medium by considering the hydraulic conductivity to be dependent on soil water content or soil matric potential. Richards (1931) combined the Darcy-Buckingham equation with the equation of continuity to provide a governing partial differential equation for transient flow in an unsaturated porous medium.

Many investigators have evaluated the Darcy and Darcy-Buckingham equations for water flow in soils of various textures (Swartzendruber, 1966). The equations were

Department of Plant and Soil Sciences, Oklahoma State Univ., Stillwater, OK 74078. Contribution of Oklahoma Agric. Exp. Stn. Received 10 Jan. 2000. *Corresponding author (dln@mail.pss.okstate.edu).

Published in J. Nat. Resour. Life Sci. Educ. 29:130-134 (2000) http://www.JNRLSE.org found to describe the flow well under many conditions. Deviations are known to exist at high flow rates due to kinetic effects or turbulence (Fancher and Lewis, 1933). Swartzendruber (1962) reported slight greater than proportional flow at much lower flow rates for mixtures of sand and clay particles when clay contents exceeded 5\%. In spite of these limitations, the flow equations of Darcy and Buckingham are still useful tools for understanding and predicting water movement in soils.

The Darcy-Buckingham equation can be written as

$$
q=-K(\theta) \frac{d H}{d x}
$$

where $q$ is the flux density of water (or the volume of water flowing per unit time per unit cross-sectional area), $K(\theta)$ is the hydraulic conductivity of the soil at volumetric water content, $\theta, H$ is the total water potential (or the sum of the gravitational and matric potentials), and $x$ is the position coordinate in the direction of flow. The quantity $(-d H / d x)$ is commonly called the driving force or hydraulic gradient. For uniform saturated soils, this equation takes the simpler form of

$$
q=K_{\mathrm{s}} \frac{H_{\text {in }}-H_{\text {out }}}{L}
$$

where $K_{\mathrm{S}}$ is the saturated hydraulic conductivity, $H_{\text {in }}$ and $H_{\text {out }}$ are the total potentials at the inlet and outlet of the soil system, respectively, and $L$ is the distance between the inlet and outlet (see Table 1 for more explanations of these terms). When the total potential, matric potential, and gravitational potential are specified on a unit-weight basis, they have units of length. The position or length of the soil system also has units of length. Therefore, the flux density and hydraulic conductivity both have units of length per unit time.

Understanding water movement in soils is essential for managing and protecting our water resources. It is important that scientists, engineers, farm managers, resource managers, and the general public understand this process. Traditional texts such as Hillel (1982, p. 57-135) introduce the concept of potential followed by mathematical equations describing flow through saturated soils. Darcy's equation is then applied to different flow geometries and algebraic expressions for the flux density are derived. Rarely will these texts derive and discuss equations for changes in potential with distance. Algebraic expressions are often shown for flow through a saturated soil made up of two layers. Following this discussion, flow in unsaturated soils is introduced using the Darcy-Buckingham equation. The impact of water content upon hydraulic conductivity is then discussed. Rarely will these texts present calculations of flux densities for unsaturated soils because the calculations can be difficult. The distribution of total, matric, and gravitational potentials with position along the soil column are also not illustrated. We have never seen these texts illustrate steady-state flow through multilayered unsaturated soils. These omissions are not due to a lack of understanding 
Table 1. Explanation of technical terms used in this manuscript.

Total potential: The amount of work that must be done per unit quantity of pure water to transport reversibly and isothermally an infinitesmal quantity of water from a pool of pure water at a specified elevation at atmospheric pressure to the soil water (Hillel, 1982, p. 66). It is also the sum of the gravitational and matric potentials.

Matric potential: The amount of work that must be done per unit quantity of pure water to transport reversibly and isothermally an infinitesmal quantity of water from a pool of pure water at the elevation of interest at atmospheric pressure to the soil water. Matric potentials are less than zero for unsaturated soils. Positive matric potentials that occur in saturated soils are sometimes called pressure potentials.

Gravitational potential: The amount of work that must be done per unit quantity of pure water in order to transport reversibly and isothermally an infinitesmal quantity of water from a pool of pure water at a specified elevation at atmospheric pressure to a pool of pure water at the elevation of interest.

Flux density of water: The volume of water flowing per unit time per unit cross-sectional area.

Hydraulic conductivity: The proportionality factor between the flux density of water and the driving force (or the gradient in total potential) in Darcy's equation or in the Darcy-Buckingham equation. That is, it is the flux density of water per unit driving force.

Equivalent conductivity: The proportionality factor in the Darcy or Darcy-Buckingham equation between the flux density of water through a soil (that may not be uniform in water content or hydraulic properties) and the overall driving force or hydraulic gradient across that entire soil.

Driving force or hydraulic gradient: A vector (macroscopic) point function that is equal to the decrease in the hydraulic head per unit distance through the soil in the direction of the greatest rate of decrease. In isotropic soils, this will be in the direction of the water flux (SSSA, 1998).

Volumetric water content: The volume of water lost from a soil per unit bulk volume of soil upon drying to constant mass at $105^{\circ} \mathrm{C}$.

Water characteristic curve: The relationship between the soil-water content (by mass or volume) and the soil-water matric potential (SSSA, 1998).

of the processes by the authors, but rather to the limitations of static printed material.

We developed interactive software for use on the World Wide Web to fill some of these voids and to enhance our understanding of steady-state flow in homogeneous and layered soil systems. The program simulates steady-state, one-dimensional water flow under saturated or unsaturated conditions. When the user accesses the software for a homogeneous system with a Java-enabled Web browser, the screen shown in Fig. 1 appears. The upper part of the screen is used to spec- ify the flow parameters. Those include the matric potential or the total water potential at each end of the soil column, the length and orientation of the soil column, and its saturated hydraulic conductivity. The lower part displays the selected graph, flux density, and equivalent conductivity. The equivalent conductivity, $K_{\mathrm{eq}}$ is defined by the equation

$$
q=K_{\mathrm{eq}} \frac{H_{\mathrm{in}}-H_{\mathrm{out}}}{L}
$$

where $q, H_{\text {in }}, H_{\text {out }}$, and $L$ are defined as in Eq. [2]. If the soil is saturated and homogeneous, $K_{\mathrm{eq}}$ is equal to $K_{\mathrm{S}}$. Otherwise, it represents the conductivity of an equivalent uniform soil with the same flow rate for the imposed driving force.

The six output options include graphs of total potential, matric potential, gravitational potential, hydraulic conductivity, driving force, and water content as functions of position along the soil system. Each time the user modifies an input parameter using the keyboard or mouse, the system solves Eq. [1] numerically and displays the output of interest. A second window (Fig. 2) is used to define additional parameters needed for unsaturated flow. These parameters define the water release or water characteristic curve and the unsaturated hydraulic conductivity function (Brooks and Corey, 1964; Gardner, 1958; Simmons et al., 1979; van Genuchten, 1980). This screen also permits the user to view graphs of the selected conductivity and water characteristic curves. Screens for layered soils are similar to those shown in Fig. 1 and 2 but with additional input provisions for the conductivity, length, and hydraulic properties of each layer.

\section{INSTRUCTIONAL USES OF SOFTWARE}

This software can be used in a wide variety of ways in an educational program. Table 2 lists some basic concepts of water flow that we consider important. The software can be used to illustrate all of these concepts. Below are a few ways in which we have used it.
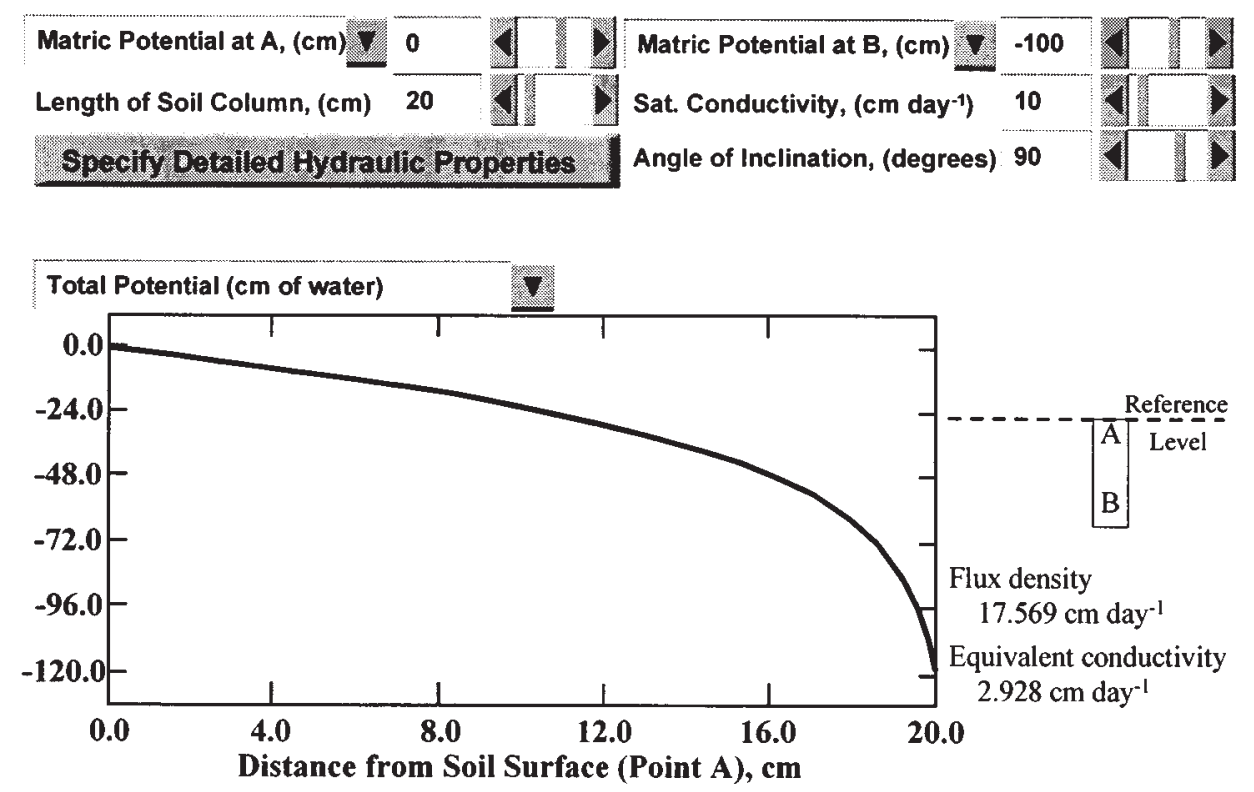

Fig. 1. View of main screen of applet for a single soil layer. 
Encourage Independent Discovery. Instructors can facilitate independent thinking and discovery by assigning exercises that utilize the software. For example, the Appendix includes exercises that were used in a senior-level soil physics class at Oklahoma State University. Before the first assignment was made, the students had been introduced to the concept of potential but no flow equations had been discussed. Exercise 1 was intended to enable them to observe the impact of different parameters upon the flux density for saturated soils. They were also asked to formulate an algebraic equation capable of predicting the flux for all of the systems used. Exercises 2 and 3 are designed to expand this understanding to more complex flow systems involving unsaturated and layered soils. Early portions of the exercises lead the student step by step through the experiment. Later portions require the student to apply what they have learned to solve new problems where details are not provided. These exercises enable students to discover flow principles on their own by assimilating information from many simulations. The exercises encourage independent thinking and analysis. They expose students to flow systems that would not be included normally because of the complexity of the solutions and time required to determine them.

Introduce and Illustrate Concepts. When lecturing with the aid of a computer and projection unit, the software can be used by the instructor to illustrate a concept. For example, the opening screen shown in Fig. 1 shows a soil with a flux density that is greater than the saturated conductivity. So an instructor can immediately ask, "How can that be?" The instructor can also quickly illustrate the impact of flow direction upon the rate of water movement. The software can also be used to introduce concepts such as hydraulic conductivity and driving force. The applet for layered soils enables the user to see the impact of changes in conductivity and thickness of a surface seal or a buried layer upon the flux density (see Exercise 3 in the Appendix). Many other examples can be given.
Table 2. Important concepts for understanding water movement in soils.

Water

a. Moves from higher total potential to lower total potential.

b. Can move upward against the force of gravity.

c. Can move from a lower matric potential to a higher matric potential.

d. Can move from lower water content to higher water content.

Potential

a. Total potential is the sum of gravitational and matric potentials (ignoring osmotic and other potentials).

b. Gravitational potential in horizontal systems is constant.

c. Total potential decreases monotonically in the direction of flow.

d. Total potential decreases linearly from the inlet to the outlet of a saturated homogeneous soil. The decrease need not be linear in unsaturated soils and in general it will not be linear.

e. Total potentials decrease in a curvilinear manner for soils in which water contents are not uniform.

f. For nonuniform systems (due to nonuniform water contents or nonuniform hydraulic properties), the change in total potential per unit distance increases as the conductivity decreases.

g. Total potentials are equal throughout a porous medium when the flow rate is zero.

h. Matric potentials and water contents can be different throughout a porous medium when flow is zero.

Flux density

a. Is proportional to hydraulic conductivity.

b. Is proportional to difference in total potential.

c. Is inversely proportional to the length of the soil.

d. Can be greater or smaller than the saturated conductivity.

e. The smaller the conductivity, the greater the required driving force to maintain the same flux density.

f. Can be larger in unsaturated soils than in saturated ones due to larger driving force.

Hydraulic conductivity

a. Is a constant for matric potentials greater than zero or saturated soils.

b. Decreases as the water content of the soil decreases.

c. Decreases as matric potential decreases for matric potentials less than zero.

d. If the conductivity of a soil changes with soil position, the equivalent conductivity is greater than the minimum conductivity in the column and less than the maximum conductivity.

Driving force

a. Does not change with position for a soil with a uniform conductivity.

b. For one-dimensional steady-state flow: If the conductivity of a soil changes with position, the driving force also changes so the flux is a constant at all positions.

Water content

a. Remains unchanged as matric potentials change for positive matric potentials.

b. Decreases as matric potential decreases for matric potentials less than zero.

c. Water can move from regions of lower water content to regions of higher water content.

\section{Specify Hydraulic Property Models and Parameters}

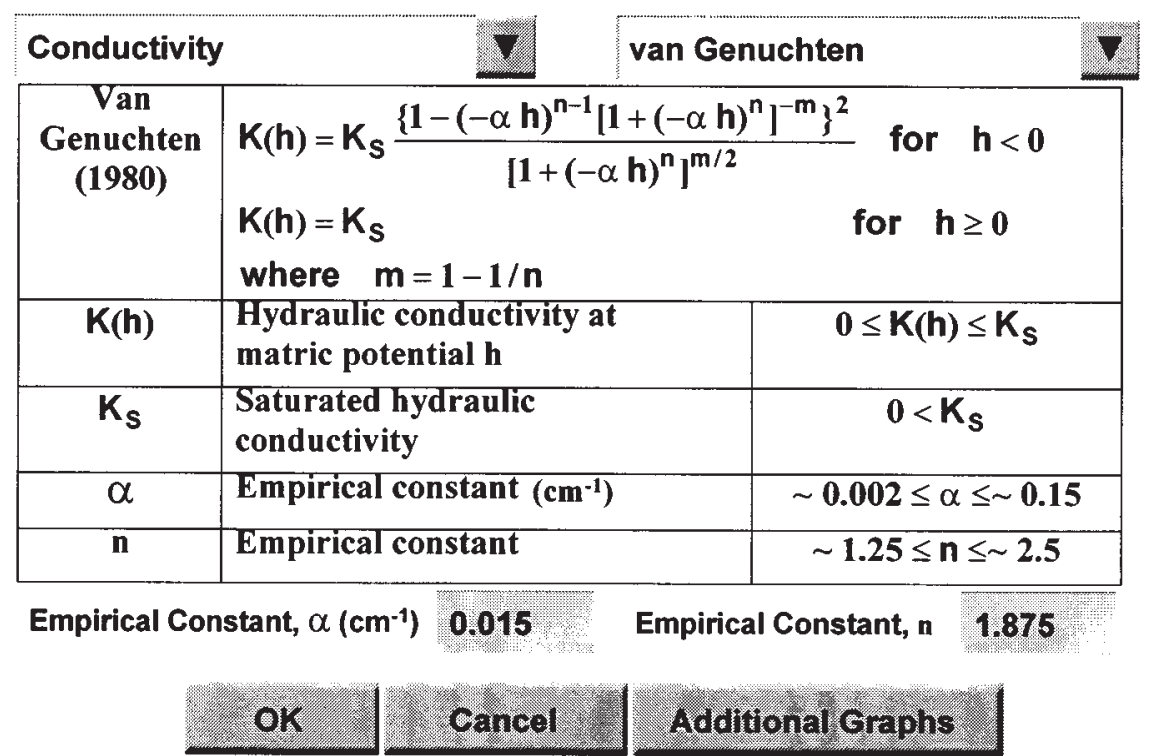

Fig. 2. Window for selecting conductivity and water characteristic models and their parameters. 
Although the applet solves the flow equation for steadystate conditions, it can be used to introduce transient flow such as infiltration. Exercise 4 describes a set of simulations used to examine the manner in which the infiltration rate changes during wetting for a soil that is initially somewhat dry. During infiltration into initially dry soils, the infiltration rate or flux density at the soil surface is equal to the product of the unsaturated hydraulic conductivity of the soil and the driving force. Initially the driving force is large and the conductivity is relatively small. As time passes, the water content of the soil increases so the conductivity increases. At the same time, the driving force decreases. This exercise enables the user to observe the net effect of these opposing factors. By plotting the flux as a function of matric potential at the bottom of the soil system, the user can see that the infiltration rate decreases with increasing matric potential, and hence it decreases with time. Although the exact shape of the function with time cannot be determined with this software, the general trend and reasons for it can be observed. This exercise enables students to build on their understanding of steady-state flow while learning the basics of transient flow.

Calculate Results for Specific Systems of Interest. The software permits the user to define the hydraulic properties and flow parameters for many different soil systems. This enables users to examine flow rates for soils of particular interest to them. These numerical experiments can simulate laboratory experiments or they may be exploratory what if exercises. Many users will not know the appropriate hydraulic parameters needed to simulate unsaturated flow in specific local soils. However, a local expert can provide this information in their local Web page used to access the applets.

Test Understanding. Instructors can use the applet to create graphs of flow systems that can be used to test comprehension of important concepts. These can be used in a lecture to encourage discussion or captured and incorporated into an examination. For example, a graph of total potential as a function of distance for an unsaturated soil can be used to determine if a student can tell the direction of water movement and explain how the conductivity changes with position.

Sensitivity Studies. The extent to which water movement changes as soil properties or flow conditions change provides useful information for assessing real world spatial or temporal differences in these properties. The applets can be used to assess this sensitivity. Users can change any input parameter and immediately observe the resulting change in flux density, equivalent conductivity, or the graph of the selected parameter. For example, a user can see how rapidly the flux density changes as the matric potential of one end becomes negative and the soil becomes unsaturated. More advanced students can change the model and parameter values used to describe the unsaturated hydraulic conductivity and water characteristic curve and observe the changes in the output. Without the ability to quickly and conveniently make these simulations, students will not be able to gain this insight.

\section{SOFTWARE SPECIFICATIONS}

The Darcy applets were written in Java 2.0. They can be run from Web browsers supporting Java applets. Best results are obtained when used with a free plug-in for the browser. The plug-in for various operating systems can be downloaded automatically from Sun Microsystems the first time it is needed. We have tested the applets with Netscape 4.0 or higher and MS Explorer 4.0 or higher. The computer must have at least $32 \mathrm{MB}$ of random access memory (64 MB recommended), and $15 \mathrm{MB}$ of free fixed disk space. The software can be accessed at http://soilphysics.okstate.edu/toolkit/. The compiled software can be downloaded and stored on a local machine so it can be used without network access. The software can also be loaded on a local Web server and incorporated into other Web pages. Contact the senior author for details.

\section{APPENDIX: NUMERICAL EXPERIMENTS}

This Appendix provides an overview of some exercises involving these applets that we used at Oklahoma State University. Detailed instructions for each exercise can be obtained at http://soilphysics.okstate.edu/toolkit/.

\section{Exercise 1: Steady-State Water Movement in Homogeneous Saturated Soils}

These experiments are designed to introduce students to water movement in uniform saturated soils. Observations to be made and questions asked are designed to enable the user to do the following:

1. See that water content is uniform throughout the soil system.

2. Interpret flux density in terms of volumetric flow rate.

3. Distinguish between flux density and hydraulic conductivity.

4. Understand the impact of changes in inlet and outlet potentials, column length, and column orientation upon flux density.

5. Observe form of potentials as functions of distance along the soil and verify that total potential is equal to the sum of the gravitational and matric potentials everywhere in the soil.

6. Discover conditions needed to obtain a flux density of zero.

7. Formulate an algebraic expression that describes all of the observations made on different flow systems. Design new experiments on which to test the expression developed.

\section{Exercise 2: Steady-State Water Movement in Unsaturated Soils}

These experiments are designed to extend the knowledge gained in Exercise 1 to include flow in unsaturated soils. In Exercise 1, matric potentials were always greater than or equal to zero. Here they are allowed to become negative and the impact of that upon flow is observed. Here the student can discover the following:

1. Soil water content decreases as matric potential becomes more and more negative.

2. As matric potentials at ends of soil column decrease (and are less than zero), the flux density decreases, even if the driving force remains the same.

3. Hydraulic conductivity decreases with decreasing matric potential in unsaturated soils.

4. Total and matric potentials are generally not linearly related to position in the soil. Users are challenged to explain why this occurs based on discoveries made already.

5. Water does not always move from high water contents to lower water contents.

6. Water content within a soil need not be uniform when no flow occurs.

\section{Exercise 3: Steady-State Water Movement in Layered Soils}

In nature, we seldom encounter uniform soils. Hydraulic properties often change with depth. It is not uncommon to have different 
hydraulic conductivities in different layers or horizons within a soil. These experiments utilize the applet for layered soils to enable the user to observe impacts of soil layers upon one-dimensional steadystate flow. Experiments are designed to enable the user to observe the following:

1. The flux density through layered soils depends on length and conductivity of each layer, not just the conductivity of the layer with the lowest conductivity.

2. For saturated soils, the driving force is constant within a layer, but greatest in the layer with lowest conductivity.

3. For saturated soils, the equivalent conductivity lies between the saturated conductivities of the two layers.

4. For saturated soils, interchanging the two layers does not affect the flux density.

5. Some aspects of flow in layered unsaturated soils are similar to those of layered saturated soils. Others are not.

6. Flow systems exist where both ends are saturated but places within may be unsaturated. The student is challenged to deduce the requirements for such a system and to test their deduction with additional experiments.

7. Flow systems exist where both ends are unsaturated but places within may be saturated. The student is challenged to deduce the requirements for such a system and to test their deduction with additional experiments.

\section{Exercise 4: An Introduction to Infiltration}

Exercises 1, 2, and 3 deal with steady-state water movement where flow rates and potentials do not change with time. In general, water movement in soils is very dynamic and changes from time to time. Infiltration is the process of water entering the soil surface. If the supply is not limiting as in the case of flood irrigation, the infil- tration rate (or flux density through the soil surface) is initially high and decreases with time. This exercise is designed to help users understand why this occurs. Thus, it forms a nice bridge from an introductory study of steady-state flow to the more complex transient flow.

\section{REFERENCES}

Brooks, R.H., and A.T. Corey. 1964. Hydraulic properties of porous media. Hydrology Pap. 3. Colorado State Univ., Ft. Collins, CO.

Buckingham, E. 1907. Studies on the movement of soil moisture. USDA Bur. Soils Bull. 38. U.S. Gov. Print. Office, Washington, DC.

Fancher, G.H., and J.A. Lewis. 1933. Flow of simple fluids through porous materials. Ind. Eng. Chem. 25:1139-1147.

Gardner, W.R. 1958. Some steady state solutions of the unsaturated moisture flow equation with application to evaporation from a water table. Soil Sci. $85: 228-232$.

Hillel, D. 1982. Introduction to soil physics. Academic Press, San Diego, CA.

Richards, L.A. 1931. Capillary conduction of liquids through porous mediums. Physics 1:318-333.

Simmons, C.S., D.R. Nielsen, and J.W. Biggar. 1979. Scaling of field-measured soil water properties. Hilgardia 47:77-173.

Slichter, C.S. 1899. Theoretical investigations of the motion of ground water. p. 295-384. In U.S. Geol. Surv. 19th Ann. Rep. 1897-98. USGS, Washington, DC.

Soil Science Society of America. 1998. Glossary of soils science terms. [Online.] Available at http://www.soils.org/sssagloss/ (verified 8 Sept. 2000).

Swartzendruber, D. 1962. Non-Darcy flow behavior in liquid-saturated porous media. J. Geophys. Res. 67:5205-5213.

Swartzendruber, D. 1966. Soil-water behavior as described by transport coefficients and functions. Adv. Agron. 18:327-370.

van Genuchten, M.Th. 1980. A closed-form equation for predicting the hydraulic conductivity of unsaturated soils. Soil Sci. Soc. Am. J. 44:892-898. 